Research Paper

\title{
Intensive Care Unit Admission and Survival in Stage IV Cancer Patients with Septic Shock: A Population-Based Cohort Study
}

\author{
Meng-Rui Lee ${ }^{1,2,3}$, Chao-Lun Lai1,2,3,4凶${ }^{\bowtie}$ K. Arnold Chan ${ }^{5,6}$ \\ 1. Department of Internal Medicine, National Taiwan University Hospital Hsin-Chu Branch, Hsin-Chu, Taiwan \\ 2. Department of Internal Medicine, College of Medicine, National Taiwan University, Taipei, Taiwan \\ 3. Institute of Epidemiology and Preventive Medicine, College of Public Health, National Taiwan University, Taipei, Taiwan \\ 4. Center for Critical Care Medicine, National Taiwan University Hospital Hsin-Chu Branch, Hsin-Chu, Taiwan \\ 5. Department of Medical Research, National Taiwan University Hospital, Taipei, Taiwan \\ 6. Graduate Institute of Oncology, College of Medicine, National Taiwan University, Taipei, Taiwan
}

$\triangle$ Corresponding author: Chao-Lun Lai, M.D., P.h.D. No.25, Lane 442, Sec. 1, Jingguo Rd., Hsinchu City 30059, Taiwan. Department of Internal Medicine, National Taiwan University Hospital Hsin-Chu Branch, Hsin-Chu, Taiwan. Tel: +886-3-5326151; Fax: +886-3-5353313; E-mail: chaolunlai@ntu.edu.tw

(C) Ivyspring International Publisher. This is an open access article distributed under the terms of the Creative Commons Attribution (CC BY-NC) license (https://creativecommons.org/licenses/by-nc/4.0/). See http://ivyspring.com/terms for full terms and conditions.

Received: 2018.09.29; Accepted: 2019.04.15; Published: 2019.06.02

\begin{abstract}
Background: The impact of intensive care unit (ICU) admission during life-threatening critical illness on survival of patients with advanced cancer remains unknown.

Methods: We identified incident stage IV cancer patients from Taiwan Cancer Registry during 2009-2013 and ascertained the first episode of septic shock after cancer diagnosis. Patient was classified as ICU admission and no ICU admission during the index hospitalization. Primary outcome of interest was overall survival. Propensity score (PS) and proportional hazards regression were used to control potential confounders.

Results: A total of 11,825 stage IV cancer patients with septic shock were identified. Among them, 6,089 (51.5\%) patients were admitted to ICU during the index hospitalization and 3,626 (30.7\%) patients survived the index hospitalization. A 1:1 propensity score (PS)-matched cohort of 7,186 patients were created for patients with/without ICU admission among the total study population. Both the PS-stratified analysis among the overall population (pooled hazard ratio [HR]: $0.78,95 \%$ confidence interval $[\mathrm{Cl}]: 0.74-0.81$ ) and analysis among the PS-matched population (HR: $0.76,95 \%$ $\mathrm{Cl}$ : 0.72-0.79) showed association between ICU admission and better overall survival. ICU admission was also associated with a lower risk of in-hospital mortality in both PS-stratified analysis (pooled odds ratio [OR]: 0.69, 95\% Cl: $0.63-0.75$ ) and PS-matched analysis (OR: $0.61,95 \% \mathrm{Cl}$ : 0.55-0.68). In PS-stratified analysis for long-term survival after discharge among hospital survivors, ICU admission was associated with improved long-term survival after discharge (pooled HR: 0.73, $95 \% \mathrm{Cl}: 0.68-0.80$ ). Also ICU admission was associated with better long-term survival after discharge (HR: 0.77, 95\% Cl: 0.70-0.85) in PS-matched analysis.

Conclusions: Though ICU admission with aggressive treatment may be associated with improved survival, the majority (70\%) of stage IV cancer patients with septic shock were unable to survive until hospital discharge.
\end{abstract}

Key words: cancer, septic shock, intensive care unit, survival

\section{Introduction}

Malignancy is an important non-communicable global disease estimated to be causing 9 million deaths annually [1]. Recent advance in anti-cancer treatment has gradually improved the overall 
survival of patients with metastatic cancer [2-4]. Despite the improvement in cancer treatment and patient survival, facing and treating critical illness such as sepsis, however, in cancer patients remains inevitable in most patients' clinical course.

Admitting to intensive care unit (ICU) is an aggressive approach for treating critical illness; however, ICU admission also means less time for family companion and invasive procedures in ICU lead to suffering and psychological trauma to patients and their caregivers $[5,6]$. ICU admission was once considered unsuitable and futile for patients with cancer diagnosis, either from a physician's or patient's perspective $[7,8]$. In a multi-center study in France, the presence of metastatic cancer was associated with nearly 6-fold increase in ICU refusal by physician, patient or family [7]. Furthermore, one ICU admission for cancer patient may deprive the opportunity of another patient to be admitted to ICU in case of limited critical care resource.

As more data are accumulating that the survival of critical cancer patients are improving, the attitude toward ICU admission for cancer patients may have gradually changed $[9,10]$. Most studies addressing cancer patients and critical care utilization, however, focused only on short term (in-hospital) outcome, were in lack of population-based data, and did not include a comparison group in which septic shock was managed outside ICU $[9,11]$.

There is general expectation of helping individual patient survive critical events and thus lead to long-term survival for physicians with an optimistic and aggressive attitude toward ICU admission [12-15]. This expectation, or hypothesis, however, has not been explored before.

\section{Material and Methods}

\section{Participants and setting}

This study was conducted with linkage of Taiwan National Health Insurance (NHI) claims data, mortality data from the Department of Statistics, and Taiwan Cancer Registry. The NHI claims data in Taiwan has been previously described $[16,17]$.

Taiwan Cancer Registry is a prospective population-based cancer data collection platform launched since 1979. In Taiwan Cancer Registry, TNM staging according to American Joint Committee on Cancer staging edition at initial diagnosis is available in the long-form database, which includes more than $90 \%$ of all cancer patients in Taiwan [18]. Through linkage between Taiwan Cancer Registry, NHI claims data, and mortality data, researchers are able to follow cancer patients from initial diagnosis, treatment course to the end of life.
We identified incident stage IV cancer (at initial diagnosis) patients in Taiwan Cancer Registry during 2009-2013 [18]. The enrolled patients were divided into two groups, namely with/without admission to ICU during their index hospitalization for septic shock.

\section{Inclusion and Exclusion Criteria}

Patients were included if they had their first episode of septic shock after diagnosis of stage IV cancer. Patients were excluded if (1) had history of admission to ICU before their first septic shock episode, (2) had history of inotropic agents use before their first septic shock episode, (3) age $<20$ years at cancer diagnosis.

\section{Definition and Data Collection}

Though ICU admission could be identified during a specific hospitalization, definite date of ICU admission during that hospitalization was not readily available in the NHI data. Cohort entry date was the date of admission of the index hospitalization for septic shock.

The diagnosis of stage IV cancer was ascertained through Taiwan Cancer Registry. Patients were defined to have septic shock if they fulfilled both the following criteria 1. The International Classification of Diseases, Ninth Revision, Clinical Modification (ICD-9-CM) codes for both a bacterial or fungal infectious process and a diagnosis of acute organ dysfunction or a ICD-9-CM code of severe sepsis (995.92) or septic shock (785.52). 2. Use of any inotropic agents, including dopamine, norepinephrine, epinephrine and vasopressin $[19,20]$.

Cancer-type was divided into 14 categories, including oral cavity, oropharynx, hypopharynx, esophagus, stomach, colon, rectum, liver, lung, breast, uterine cervix, prostate, bladder and other cancer. The designation of International Classification of Diseases for Oncology, 3rd edition (ICD-O-3) codes for each cancer type was illustrated in Table S1. We used the Deyo version of the Charlson comorbidity index (CCI) for assessing patients' underlying medical condition and calculated the CCI according to medical claims records in NHI claims database one year before cohort entry [21]. Socioeconomic status was determined by income reported for premium calculation, which was divided into low income (receiving government subsidies due to being below the lowest living index and being exempted from NHI premiums and copayment), $\leqq \mathrm{Q} 1, \mathrm{Q} 1$ to $\mathrm{Q} 3$ and $\geqq \mathrm{Q} 3$ as previously published $[22,23]$. The codes for variable definitions used in this study were summarized in Table S2. 


\section{Statistical analysis}

Proportions or means were used to describe the demographic and clinical characteristics of the patients. Standardized difference was used to compare between continuous variables and categorical variables at baseline before the index hospitalization. The propensity score (PS) for the probability of being admitted to ICU was derived using logistic regression model including potential confounders such as age, sex, socioeconomic status, cancer type, use of positron emission tomography (PET), CCI, antineoplastic therapy, interval between cancer diagnosis and the occurrence of septic shock, and inotropic agents used.

Primary outcome was overall survival, which was defined as the interval between date of admission of the index hospitalization and date of death. Secondary outcomes included in-hospital mortality and long-term survival after discharge from the index hospitalization which was defined as the interval between date of discharge and date of death. Participants were censored if they were still alive at end of the study period (2014-12-31).

For analysis of overall survival, we included all stage IV cancer patients experiencing their first septic shock episode after the initial diagnosis. The proportional hazards regression model stratified on quintiles of the PS was applied to compare the overall survival between patients with/without ICU admission as the PS-stratified analysis. As for the PS-matched analysis, a 1:1 PS-matched population was created according to caliper measurements of < 0.2 standard deviations of the logit of the PS for stage IV cancer patients admitted and not admitted to the ICU. And the proportional hazards regression model was applied to estimate the relative hazard of death between patients with/without ICU admission among the PS-matched population.

Concerning in-hospital mortality, the PS-stratified analysis was conducted by means of the logistic regression model to estimate the odds ratio (OR) within each of the PS quintile stratum with the pooled OR across 5 PS strata obtained by the Mantel-Haenszel estimate. Besides, the logistic regression model was also used among the PS-matched population for analysis of in-hospital mortality.

For long-term survival after discharge, we included only patients who survived to discharge from the index hospitalization with another set of PS created accordingly.

A sensitivity analysis was performed with exclusion of patients who died within 7 days after hospital discharge for evaluation of long-term survival after discharge. Subgroup analyses were performed with stratification by specific cancer types. All data analyses were performed using SAS version 9.4 (SAS Institute Inc, Cary, NC, USA). A P $<0.05$ on a two-sided test or a standardized difference $>0.1$ was considered statistically significant.

\section{Results}

A total of 11,825 stage IV cancer patients fulfilled the inclusion criteria. The patient identification process is illustrated in Fig. 1. Clinical characteristics of stage IV cancer patients experiencing septic shock are illustrated in Table 1. Among them, 6,089 (51.5\%) patients were admitted to ICU during the index hospitalization while 5,736 (48.5\%) patients were not admitted to ICU during the index hospitalization. The mean age was 66 and there was a male preponderance (M/F: $8,465 / 3,360)$. The most common cancer type was lung cancer $(n=4,097$, $34.6 \%)$, followed by liver cancer $(n=1,046,8.8 \%)$ and oral cancer $(n=1,046,8.8 \%)$. More than half $(n=6,313$, $53.4 \%$ ) patients received chemotherapy before this index hospitalization. Those admitted to ICU were more likely to be male. Those admitted to ICU were more likely to have oral cavity cancer and less likely to have liver cancer. Patients with ICU admission were more likely to receive PET and various vasopressors than those not admitted to ICU. The crude in-hospital mortality rate among the overall population was $69 \%(68.7 \%$ in the ICU admission group versus $70 \%$ in the ICU no admission group). The medical utilizations including artificial organ support, cardiopulmonary resuscitation (CPR), and palliative care in the study population were summarized in Table S3.

A 1:1 PS-matched cohort of 7,186 patients for analyzing overall survival and in-hospital mortality were created. After matching, the baseline characteristics were similar between two groups (Table 1).

ICU admission was associated with better overall survival in both PS-stratified analysis (pooled HR: 0.78, 95\% CI: 0.74-0.81, Table 2) and PS-matched analysis (adjusted HR: 0.76, 95\% CI: 0.72-0.79). In addition, ICU admission was associated with a lower risk of in-hospital mortality in both PS-stratified analysis (pooled OR: 0.69, 95\% CI: 0.63-0.75) and PS-matched analysis (OR: 0.61, 95\% CI: 0.55-0.68, Table S4).

A total of 3,626 (30.7\%) patients survived to hospital discharge. The most common cancer type among hospital survivors were lung cancer $(\mathrm{n}=996$, $27.5 \%)$ and oral cancer $(\mathrm{n}=633,17.5 \%)$. Among the 3,626 hospital survivors, 1,908 (52.6\%) patients have been admitted to ICU during hospitalization (Table 3). The medical utilizations including artificial organ 
support, cardiopulmonary resuscitation and palliative care in the study population who survived to discharge from the index hospitalization were summarized in Table S5.

Another 1:1 PS-matched cohort of 2,194 patients were created among survivors of the index hospitalization. After matching, all baseline characteristics became balanced between groups except for oral cancer (oral cancer was more common among those not admitted to ICU compared with those admitted to ICU, $15.0 \%$ vs $10.9 \%$, standardized difference $=0.122$ ) (Table 3). In PS-stratified analysis for long-term survival after discharge among hospital survivors, ICU admission was associated with improved long-term survival (pooled HR: 0.73, 95\% CI: 0.68-0.80) (Table S6). Also ICU admission was associated with better long-term survival after discharge (HR: 0.77, 95\% CI: 0.70-0.85) in PS-matched analysis.

Forest plots regarding association between overall survival and ICU admission stratified by different cancer types are illustrated in Fig. 2. A sensitivity analysis was performed for analysis of long-term survival after discharge among hospital survivors by excluding patients who died within 7 days after discharge from the index hospitalization. The results of stratified PS analysis were similar to our original analysis and all indicated an association between ICU admission and better long-term survival after discharge. ICU admission was associated with better long-term survival after discharge (HR: 0.85, 95\% CI: 0.76-0.94) in PS-matched analysis. The detailed results of sensitivity analysis is shown in Table S7 and Table S8.

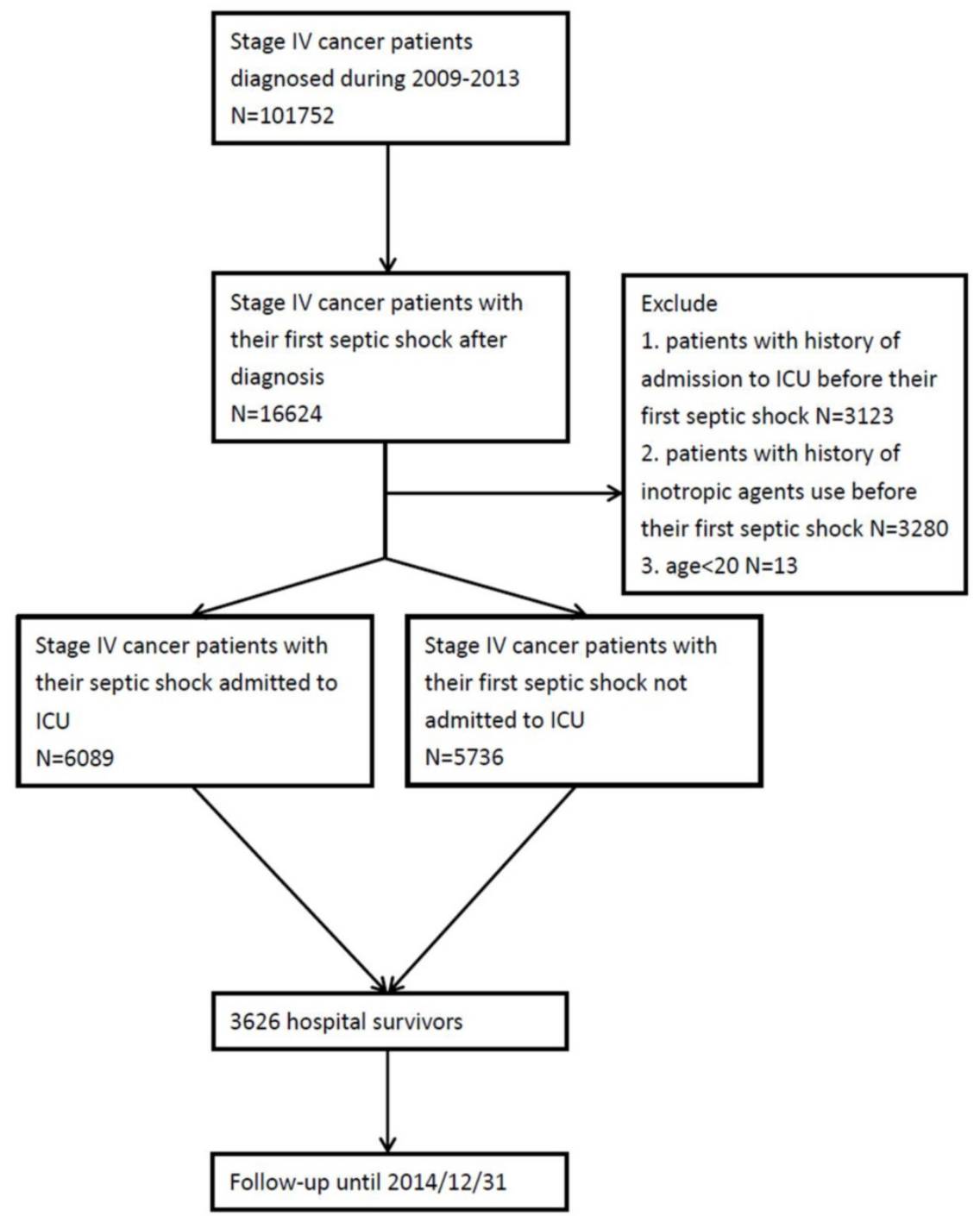

Figure 1. Patient recruitment process. Abbreviation: ICU, intensive care unit. 
Table 1. Clinical characteristics of stage IV cancer patients with septic shock.

\begin{tabular}{|c|c|c|c|c|c|c|c|c|}
\hline & \multicolumn{4}{|l|}{ Before PS Matching } & \multicolumn{4}{|c|}{ After PS Matching } \\
\hline & Overall Patients $(n=11825)$ & $\begin{array}{l}\text { No ICU admission } \\
(\mathrm{n}=5736)\end{array}$ & $\begin{array}{l}\text { ICU admission } \\
(\mathrm{n}=6089)\end{array}$ & STD & $\begin{array}{l}\text { Overall } \\
\text { Patients } \\
(\mathrm{n}=7186)\end{array}$ & $\begin{array}{l}\text { No ICU } \\
\text { admission } \\
(\mathrm{n}=3593)\end{array}$ & $\begin{array}{l}\text { ICU } \\
\text { admission } \\
(\mathrm{n}=3593)\end{array}$ & STD \\
\hline Age $($ mean $\pm S D)$ & $66.1 \pm 13.9$ & $66.8 \pm 14.1$ & $65.5 \pm 13.7$ & 0.088 & $66.3 \pm 14.0$ & $66.0 \pm 14.2$ & $66.6 \pm 13.7$ & 0.041 \\
\hline \multicolumn{9}{|l|}{ Gender } \\
\hline Male & $8465(71.6)$ & 3919 (68.3) & $4546(74.7)$ & 0.141 & $5165(71.9)$ & $2578(71.8)$ & $2587(72.0)$ & 0.006 \\
\hline Female & $3360(28.4)$ & $1817(31.7)$ & $1543(25.3)$ & & $2021(28.1)$ & $1015(28.2)$ & $1006(28.0)$ & \\
\hline \multicolumn{9}{|l|}{ Socioeconomic status } \\
\hline Low income & $291(2.5)$ & $153(2.7)$ & $138(2.3)$ & 0.026 & 187(2.6) & $95(2.6)$ & $92(2.6)$ & 0.005 \\
\hline$\leqq \mathrm{Q} 1$ & $4089(34.6)$ & $2016(35.1)$ & $2073(34.0)$ & 0.023 & $2434(33.9)$ & $1201(33.4)$ & $1233(34.3)$ & 0.019 \\
\hline Q1-Q3 & $4938(41.8)$ & $2355(41.1)$ & $2583(42.4)$ & 0.028 & $3058(42.6)$ & $1527(42.5)$ & $1531(42.6)$ & 0.002 \\
\hline$>\mathrm{Q} 3$ & $2507(21.2)$ & $1212(21.1)$ & $1295(21.3)$ & 0.003 & $1507(21.0)$ & $770(21.4)$ & $737(20.5)$ & 0.023 \\
\hline \multicolumn{9}{|l|}{ Cancer type } \\
\hline Oral Cavity & $1046(8.8)$ & $323(5.6)$ & $723(11.9)$ & 0.222 & $626(8.7)$ & $321(8.9)$ & $305(8.5)$ & 0.016 \\
\hline Oropharynx & $534(4.5)$ & $231(4.0)$ & $303(5.0)$ & 0.046 & $331(4.6)$ & $160(4.5)$ & $171(4.8)$ & 0.015 \\
\hline Hypopharynx & $440(3.7)$ & $186(3.2)$ & $254(4.2)$ & 0.049 & 261(3.6) & $133(3.7)$ & $128(3.6)$ & 0.007 \\
\hline Esophagus & 404(3.4) & $164(2.9)$ & $240(3.9)$ & 0.060 & 265(3.7) & $137(3.8)$ & $128(3.6)$ & 0.013 \\
\hline Stomach & $749(6.3)$ & $425(7.4)$ & $324(5.3)$ & 0.086 & $454(6.3)$ & $234(6.5)$ & $220(6.1)$ & 0.016 \\
\hline Colon & $878(7.4)$ & $420(7.3)$ & $458(7.5)$ & 0.008 & $556(7.7)$ & $280(7.8)$ & $276(7.7)$ & 0.004 \\
\hline Rectum & $475(4.0)$ & 221 (3.9) & $254(4.2)$ & 0.016 & $299(4.2)$ & 149 (4.1) & $150(4.2)$ & 0.001 \\
\hline Liver & $1046(8.8)$ & $615(10.7)$ & $431(7.1)$ & 0.128 & $603(8.4)$ & $309(8.6)$ & $294(8.2)$ & 0.015 \\
\hline Lung & 4097(34.6) & $2111(36.8)$ & 1986 (32.6) & 0.088 & $2467(34.3)$ & $1218(33.9)$ & $1249(34.8)$ & 0.018 \\
\hline Breast & $266(2.2)$ & $142(2.5)$ & $124(2.0)$ & 0.030 & $152(2.1)$ & $77(2.1)$ & $75(2.1)$ & 0.004 \\
\hline Cervix & $160(1.4)$ & $98(1.7)$ & $62(1.0)$ & 0.060 & $92(1.3)$ & $47(1.3)$ & 45 (1.3) & 0.005 \\
\hline Prostate & $605(5.1)$ & $272(4.7)$ & $333(5.5)$ & 0.033 & $389(5.4)$ & $187(5.2)$ & $202(5.6)$ & 0.018 \\
\hline Bladder & $169(1.4)$ & $76(1.3)$ & $93(1.5)$ & 0.017 & $107(1.5)$ & $50(1.4)$ & $57(1.6)$ & 0.016 \\
\hline Other & $956(8.1)$ & $452(7.9)$ & $504(8.3)$ & 0.015 & $584(8.1)$ & $291(8.1)$ & $293(8.2)$ & 0.002 \\
\hline Use of PET & $1175(9.9)$ & $476(8.3)$ & $699(11.5)$ & 0.107 & $702(9.8)$ & $337(9.4)$ & $365(10.2)$ & 0.026 \\
\hline $\mathrm{CCI}($ mean $\pm \mathrm{SD})$ & $9.27 \pm 1.45$ & $9.26 \pm 1.42$ & $9.27 \pm 1.47$ & 0.003 & $9.28 \pm 1.47$ & $9.27 \pm 1.44$ & $9.30 \pm 1.49$ & 0.017 \\
\hline \multicolumn{9}{|l|}{ Anti-cancer therapy } \\
\hline Chemotherapy & $6313(53.4)$ & $3042(53.0)$ & 3271 (53.7) & 0.014 & $3845(53.5)$ & $1948(54.2)$ & $1897(52.8)$ & 0.028 \\
\hline Radiotherapy & $3788(32.0)$ & $1770(30.9)$ & 2018 (33.1) & 0.049 & $2317(32.2)$ & $1167(32.5)$ & $1150(32.0)$ & 0.010 \\
\hline TKI & $1163(9.8)$ & $603(10.5)$ & $560(9.2)$ & 0.044 & $709(9.9)$ & $361(10.0)$ & $348(9.7)$ & 0.012 \\
\hline Antibody & $747(6.3)$ & $368(6.4)$ & $379(6.2)$ & 0.008 & $457(6.4)$ & $228(6.3)$ & $229(6.4)$ & 0.001 \\
\hline $\begin{array}{l}\text { Interval Between Index Hospitalization } \\
\text { Admission and Cancer Diagnosis (mean } \pm S D \text { ) }\end{array}$ & $232.7 \pm 296.5$ & $241.6 \pm 303.8$ & $224.4 \pm 289.2$ & 0.058 & $\begin{array}{l}234.2 \pm 294 \\
8\end{array}$ & $233.8 \pm 289.4$ & $234.7 \pm 300.1$ & 0.003 \\
\hline \multicolumn{9}{|l|}{ Inotropic Agents } \\
\hline Epinephrine & $6,650(56.2)$ & $3080(53.7)$ & $3570(58.6)$ & 0.100 & $3673(51.1)$ & $1845(51.3)$ & $1828(50.9)$ & 0.009 \\
\hline Dopamine & $6038(51.1)$ & $2545(44.4)$ & $3493(57.4)$ & 0.262 & $3384(47.1)$ & $1687(47.0)$ & $1697(47.2)$ & 0.006 \\
\hline Norepinephrine & $5319(45.0)$ & $1595(27.8)$ & $3724(61.2)$ & 0.712 & $2948(41.0)$ & $1464(40.7)$ & $1484(41.3)$ & 0.011 \\
\hline Vasopressin & $281(2.4)$ & $17(0.3)$ & $264(4.3)$ & 0.271 & $39(0.5)$ & $17(0.5)$ & $22(0.6)$ & 0.019 \\
\hline In-hospital mortality & $8199(69.3)$ & $4018(70.0)$ & $4181(68.7)$ & $0.103^{*}$ & $4863(67.7)$ & $2610(72.6)$ & $2253(62.7)$ & $<0.001^{*}$ \\
\hline
\end{tabular}

Table 2. Association between ICU admission and overall survival evaluated by proportional hazards model with stratification on quintiles of the propensity score.

\begin{tabular}{|c|c|c|c|c|c|c|c|}
\hline \multirow[t]{2}{*}{ Quintiles of PS } & \multicolumn{3}{|c|}{ Patients without admission to ICU } & \multicolumn{4}{|l|}{ Patients with admission to ICU } \\
\hline & Mortality rate (/person-day) & Death & Follow-up person-day & Mortality rate (/person-day) & Death & Follow-up person-day & $\mathrm{HR}(95 \% \mathrm{CI})$ \\
\hline 1 & 0.018 & 339 & 18865 & 0.012 & 1949 & 161155 & $0.78(0.70-0.88)$ \\
\hline 2 & 0.009 & 714 & 80916 & 0.004 & 1405 & 364629 & $0.67(0.62-0.74)$ \\
\hline 3 & 0.012 & 1217 & 103436 & 0.007 & 1010 & 136080 & $0.78(0.72-0.85)$ \\
\hline 4 & 0.008 & 1486 & 178014 & 0.005 & 740 & 139192 & $0.79(0.72-0.86)$ \\
\hline 5 & 0.010 & 1708 & 177186 & 0.008 & 532 & 63873 & $0.90(0.81-0.99)$ \\
\hline Pooled & & & & & & & $0.78(0.74-0.81)$ \\
\hline
\end{tabular}

Abbreviations: CI, confidence interval; HR, hazard ratio; ICU, intensive care unit; PS, propensity score

\section{Discussion}

In our study, by identifying a large population-based cohort we found that admission to ICU for septic shock was associated with better overall survival, lower in-hospital mortality and better long-term survival after discharge among stage IV cancer patients. $70 \%$ of stage IV cancer patients with septic shock, however, failed to survive until discharge. 
Table 3. Clinical characteristics of stage IV cancer patients with septic shock who survived to discharge.

\begin{tabular}{|c|c|c|c|c|c|c|c|c|}
\hline & \multicolumn{4}{|c|}{ Before PS Matching } & \multicolumn{4}{|c|}{ After PS Matching } \\
\hline & Overall $(n=3626)$ & $\begin{array}{l}\text { No ICU admission } \\
(n=1718)\end{array}$ & $\begin{array}{l}\text { ICU admission } \\
(\mathrm{n}=1908)\end{array}$ & STD & $\begin{array}{l}\text { Overall } \\
(\mathrm{n}=2194)\end{array}$ & $\begin{array}{l}\text { No ICU admission } \\
(\mathrm{n}=1097)\end{array}$ & ICU admission $(\mathrm{n}=1097)$ & STD \\
\hline Age (mean $\pm S D)$ & $64.1 \pm 13.7$ & $64.4 \pm 13.9$ & $63.7 \pm 13.5$ & 0.050 & $64.7 \pm 13.8$ & $64.4 \pm 14.0$ & $65.0 \pm 14.0$ & 0.038 \\
\hline \multicolumn{9}{|l|}{ Gender } \\
\hline Male & $2661(73.4)$ & $1189(69.2)$ & 1472(77.1) & 0.180 & $1590(72.5)$ & $800(72.9)$ & $790(72.0)$ & 0.020 \\
\hline Female & $965(26.6)$ & $529(30.8)$ & $436(22.9)$ & & $604(27.5)$ & $297(27.1)$ & $307(28.0)$ & \\
\hline \multicolumn{9}{|l|}{ Socioeconomic status } \\
\hline Low income & $94(2.6)$ & $58(3.4)$ & $36(1.9)$ & 0.093 & $60(2.7)$ & $30(2.7)$ & $30(2.7)$ & 0.000 \\
\hline$\leqq \mathrm{Q} 1$ & $1130(31.2)$ & $532(31.0)$ & $598(31.3)$ & 0.008 & $678(30.9)$ & $337(30.7)$ & $341(31.1)$ & 0.008 \\
\hline Q1-Q3 & $1659(45.8)$ & $771(44.9)$ & $888(46.5)$ & 0.033 & $1017(46.4)$ & $507(46.2)$ & $510(46.5)$ & 0.005 \\
\hline$>Q 3$ & $743(20.5)$ & $357(20.8)$ & $386(20.2)$ & 0.014 & $439(20.0)$ & $223(20.3)$ & $216(19.7)$ & 0.016 \\
\hline \multicolumn{9}{|l|}{ Cancer type } \\
\hline Oral Cavity & $633(17.5)$ & $169(9.8)$ & $464(24.3)$ & 0.394 & $285(13.0)$ & $165(15.0)$ & $120(10.9)$ & 0.122 \\
\hline Oropharynx & $241(6.6)$ & $110(6.4)$ & $131(6.9)$ & 0.019 & 155 (7.1) & $78(7.1)$ & $77(7.0)$ & 0.004 \\
\hline Hypopharynx & $211(5.8)$ & $110(6.4)$ & 101(5.3) & 0.047 & $137(6.2)$ & $68(6.2)$ & $69(6.3)$ & 0.004 \\
\hline Esophagus & $101(2.8)$ & $36(2.1)$ & $65(3.4)$ & 0.08 & $70(3.2)$ & $33(3.0)$ & $37(3.4)$ & 0.021 \\
\hline Stomach & $153(4.2)$ & $82(4.8)$ & $71(3.7)$ & 0.052 & $103(4.7)$ & $51(4.6)$ & $52(4.7)$ & 0.004 \\
\hline Colon & $255(7.0)$ & $110(6.4)$ & $145(7.6)$ & 0.047 & $166(7.6)$ & $83(7.6)$ & $83(7.6)$ & 0.000 \\
\hline Rectum & $146(4.0)$ & $61(3.6)$ & $85(4.5)$ & 0.046 & $111(5.1)$ & $54(4.9)$ & $57(5.2)$ & 0.012 \\
\hline Liver & $213(5.9)$ & $125(7.3)$ & $88(4.6)$ & 0.113 & $147(6.7)$ & $71(6.5)$ & $76(6.9)$ & 0.018 \\
\hline Lung & $996(27.5)$ & $572(33.3)$ & $424(22.2)$ & 0.249 & $587(26.8)$ & $281(25.6)$ & $306(27.9)$ & 0.051 \\
\hline Breast & $89(2.5)$ & $49(2.9)$ & $40(2.1)$ & 0.049 & $52(2.4)$ & $24(2.2)$ & $28(2.6)$ & 0.024 \\
\hline Cervix & $55(1.5)$ & $33(1.9)$ & $22(1.2)$ & 0.062 & $31(1.4)$ & $15(1.4)$ & $16(1.5)$ & 0.008 \\
\hline Prostate & $189(5.2)$ & $91(5.3)$ & $98(5.1)$ & 0.007 & $131(6.0)$ & $64(5.8)$ & $67(6.1)$ & 0.012 \\
\hline Bladder & $50(1.4)$ & $20(1.2)$ & $30(1.6)$ & 0.035 & $34(1.5)$ & $18(1.6)$ & $16(1.5)$ & 0.015 \\
\hline Other & $294(8.1)$ & $150(8.7)$ & $144(7.5)$ & 0.043 & $185(8.4)$ & $92(8.4)$ & $93(8.5)$ & 0.003 \\
\hline Use of PET & $365(10.1)$ & $149(8.7)$ & $216(11.3)$ & 0.088 & $215(9.8)$ & $108(9.8)$ & $107(9.8)$ & 0.003 \\
\hline CCI & $9.1 \pm 1.4$ & $9.1 \pm 1.4$ & $9.1 \pm 1.4$ & 0.020 & $9.1 \pm 1.4$ & $9.1 \pm 1.4$ & $9.2 \pm 2.0$ & 0.044 \\
\hline \multicolumn{9}{|l|}{ Anti-cancer therapy } \\
\hline Chemotherapy & $1668(46.0)$ & $807(47.0)$ & $861(45.1)$ & 0.037 & $1103(50.3)$ & $551(50.2)$ & $552(50.3)$ & 0.002 \\
\hline Radiotherapy & $1018(28.1)$ & $493(28.7)$ & $525(27.5)$ & 0.029 & $656(29.9)$ & $330(30.1)$ & $326(29.7)$ & 0.008 \\
\hline TKI & $227(6.3)$ & $119(6.9)$ & $108(5.7)$ & 0.052 & $151(6.9)$ & $78(7.1)$ & $73(6.7)$ & 0.018 \\
\hline Antibody & $224(6.2)$ & $104(6.1)$ & $120(6.3)$ & 0.010 & $139(6.3)$ & $70(6.4)$ & $69(6.3)$ & 0.004 \\
\hline $\begin{array}{l}\text { Interval Between Admission to ICU } \\
\text { and Cancer Diagnosis (mean } \pm \text { SD) }\end{array}$ & $206.2 \pm 298.8$ & $217.1 \pm 311.7$ & $196.3 \pm 286.4$ & 0.069 & $222.3 \pm 304.0$ & $216.2 \pm 293.7$ & $228.4 \pm 313.8$ & 0.040 \\
\hline \multicolumn{9}{|l|}{ Inotropic Agents } \\
\hline Epinephrine & 1982(54.7) & $1020(59.4)$ & $962(50.4)$ & 0.181 & $1024(46.7)$ & $519(47.3)$ & $505(46.0)$ & 0.026 \\
\hline Dopamine & 1367(37.7) & $534(31.1)$ & $833(43.7)$ & 0.262 & $879(40.1)$ & $423(38.6)$ & $456(41.6)$ & 0.061 \\
\hline Norepinephrine & $1136(31.3)$ & $308(17.9)$ & $828(43.4)$ & 0.575 & $629(28.7)$ & $300(27.3)$ & $329(30.0)$ & 0.058 \\
\hline Vasopressin & $38(1.0)$ & $9(0.5)$ & $29(1.5)$ & 0.099 & $13(0.6)$ & $7(0.6)$ & $6(0.5)$ & 0.012 \\
\hline
\end{tabular}

Abbreviations: CCI, Charlson comorbidity index; ICU, intensive care unit; PET, positron emission tomography; PS, propensity score; Q1, first quartile; Q3, third quartile; SD, standard deviation; STD, standardized difference; TKI, tyrosine kinase inhibitor.

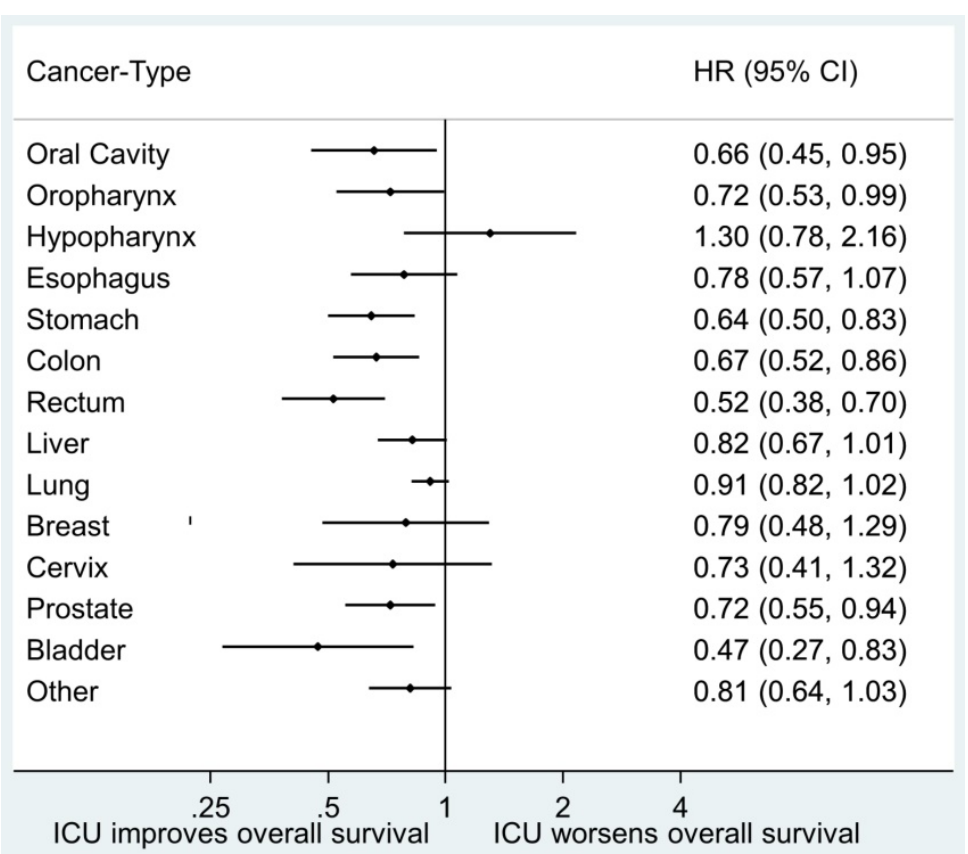

Figure 2. Forest plot of hazard ratios regarding association between ICU admission and overall survival stratified by different cancer types. Abbreviations: $\mathrm{Cl}$, confidence interval; $\mathrm{HR}$, hazard ratio, ICU, intensive care unit. 
One major strength of our study was that we investigated the impact of ICU admission on overall survival of cancer patients and included a comparison group without ICU admission, which was not commonly assessed in previous studies [9, 10, 24, 25]. In our analysis of hospital survivors of cancer patients from septic shock, an improved long-term survival after discharge was observed among those who were admitted to ICU, indicating that benefit of admitting to ICU extended beyond hospitalization. Aggressive treatment and even implementation of artificial organ during ICU care (Table S3 and Table S5) may not only improve short-term (in-hospital) survival but also help preserve organ function and thus lead to better long-term survival. For instance, in severe sepsis patients with respiratory failure, mechanical ventilation could improve gas exchange, decrease work of breathing, avoid lung damage and could be life-saving [26]. Protocolized and detailed sepsis bundle management is less likely to be implemented outside ICU setting where higher staff to patient ratio is equipped [27]. Performing and adhering to sepsis bundle improve patient survival and decrease mortality [28]. Furthermore, new insight into artificial organ support for organ protection including lung protective strategy and permissive hypercapnia may influence inflammatory mediators and preserve end-organ function [29]. The abovementioned mechanisms may together contribute to a better outcome in cancer patients with septic shock.

One may question that the decision to admit stage IV cancer patients to ICU may not be at random and may be confounded by indication. For instance, physicians may choose those who were likely to survive the event and had better anticipated anti-cancer treatment to be admitted to ICU. Indeed, not all sepsis patients were managed in the ICU [30]. Sepsis patients managed outside ICU, were found to have a lower disease severity compared with those managed in ICU [28]. The high percentage of patients receiving mechanical ventilation (76\%) in ICU in this study also indicated higher disease severity since respiratory failure is an indicator of disease severity [31]. Furthermore, the costs of ICU admission are relatively low in Taiwan and there are no restrictions on admitting cancer patients to ICU by Taiwan NHI $[32,33]$. The decision of ICU admission, therefore, was most likely to be based on patients' as well as caregivers' aggressiveness (eg, deciding to receive endotracheal intubation for respiratory failure). In our study more patients admitted to ICU received CPR and more patients not admitted to ICU received palliative care (Table S3 and Table S4). Since ICU admission was considered an aggressive attitude toward treatment, our study was in concordance with this concept [25].

The in-hospital mortality rate of septic shock patients is estimated to range from $18 \%$ to $50 \%$ [34, 35]. Though our study did not include a control group of septic shock patients without cancer, the in-hospital mortality rate (around $70 \%$ ) in our study seemed to be higher compared with previous reports [34,35]. This finding may not be surprising since we selected a group of stage IV cancer patients who were considered to have a dismal prognosis. This high in-hospital mortality rate also raises concerns of critical care service rationing and issue of equity [36, 37]. When physicians decide to admit cancer patients to ICU, it should be kept in mind that the admitted cancer patients should be likely to benefit from ICU service; otherwise this may do harm to other patients who also need critical care service.

Our study also has limitations. First, though we have implemented PS matching to control for potential confounders and included important variables including cancer-type, use of PET in staging (which was known as an indicator of stage migration and better prognostic factor) [38] and previous anti-cancer treatment in the PS, we still cannot exclude the possibility of uncontrolled confounding, which could not be ascertained from the insurance claims. Second, we have no data on quality of life among critical illness survivors. Potential deterioration in quality of life even after surviving critical illness, therefore, should be informed to family and patient [39]. Third, we were unable to recruit another independent validation cohort in Taiwan since NHI is a single-payer health system that provides universal coverage for medical services in Taiwan [40]. Further studies from different healthcare systems may be warranted to further validate our findings.

\section{Conclusions}

In conclusion, we found that utilization of ICU during septic shock in stage IV cancer patients may be associated with improved survival. Nonetheless, the in-hospital mortality rate remained high even after aggressive treatment under ICU setting. Our findings could be informative to physicians, cancer patients, and their relatives. Future studies should be aimed at providing long-term quality of life data among cancer patients surviving septic shock.

\section{Abbreviations}

CCI: Charlson comorbidity index; CPR: cardiopulmonary resuscitation; ICD-O-3: International Classification of Diseases for Oncology, 3rd edition; ICD-9-CM: International Classification of Diseases, Ninth Revision, Clinical Modification; ICU: 
intensive care unit; NHI: National Health Insurance; OR: odds ratio; PET: positron emission tomography; PS: propensity score.

\section{Supplementary Material}

Supplementary tables.

http://www.jcancer.org/v10p3179s1.pdf

\section{Acknowledgements}

We thanked Professor Yu Ming-Chih, Wang Hao-Chien and $\mathrm{Tu} \mathrm{Yu}-\mathrm{Kang}$ for suggestions on the study design and data analysis.

\section{Availability of data and materials}

All the data used in this study were released and approved by the Health and Welfare Data Science Center (HWDC), Ministry of Health and Welfare, Executive Yuan, Taiwan.

\section{Ethics Statement}

The Institutional Review Board of National Taiwan University Hospital Hsin-Chu Branch approved the study (NTUH-HC REC: 104-080-E).

\section{Author Contributions}

Conception and Design: Meng-Rui, Lee and K. Arnold Chan.

Collection and assembly of data: Meng-Rui, Lee and Chao-Lun, Lai.

Data analysis and interpretation: All authors.

Manuscript writing: All authors.

Final approval of manuscript: All authors.

Accountable for all aspects of the work: All authors.

\section{Funding source}

This study was funded by National Taiwan University Hospital Hsin-Chu Branch (105-HCH001). The funder has no role in the study design, data analysis, and manuscript writing.

\section{Competing Interests}

The authors have declared that no competing interest exists.

\section{References}

1. GBD 2015 Mortality and Causes of Death Collaborators. Global, regional, and national life expectancy, all-cause mortality, and cause-specific mortality for 249 causes of death, 1980-2015: a systematic analysis for the Global Burden of Disease Study 2015. Lancet. 2016;388:1459-544.

2. Temel JS, Greer JA, Muzikansky A, Gallagher ER, Admane S, Jackson VA, et al. Early palliative care for patients with metastatic non-small-cell lung cancer. N Engl J Med. 2010; 363:733-42.

3. Mokdad AA, Singal AG, Yopp AC: Advances in Local and Systemic Therapies for Hepatocellular Cancer. Curr Onco Rep. 2016; 18:9

4. Van Cutsem E, Sagaert X, Topal B, Haustermans K, Prenen H. Gastric cancer. Lancet. 2016:388:2654-64.

5. Cameron JI, Chu LM, Matte A, Tomlinson G, Chan L, Thomas C, et al. RECOVER Program Investigators (Phase 1: towards RECOVER); Canadian Critical Care Trials Group One-Year Outcomes in Caregivers of Critically Ill Patients. N Engl J Med. 2016;374:1831-41
6. Jutte JE, Erb CT, Jackson JC. Physical, Cognitive, and Psychological Disability Following Critical Illness: What Is the Risk? Semin Respir Crit Care Med. 2015;36:943-58.

7. Garrouste-Orgeas M, Montuclard L, Timsit JF, Reignier J, Desmettre T, Karoubi $\mathrm{P}$, et al. French ADMISSIONREA Study Group. Predictors of intensive care unit refusal in French intensive care units: a multiple-center study. Crit Care Med. 2005;33:750-5.

8. Yun YH, Lee MK, Kim SY, Lee WJ, Jung KH, Do YR, et al. Impact of awareness of terminal illness and use of palliative care or intensive care unit on the survival of terminally ill patients with cancer: prospective cohort study. J Clin Oncol. 2011;29:2474-80

9. Pène F, Percheron S, Lemiale V, Viallon V, Claessens YE, Marqué S, et al. Temporal changes in management and outcome of septic shock in patients with malignancies in the intensive care unit. Crit Care Med. 2008;36:690-6.

10. Legrand M, Max A, Peigne V, Mariotte E, Canet E, Debrumetz A, et al. Survival in neutropenic patients with severe sepsis or septic shock. Crit Care Med. 2012;40:43-9.

11. Ostermann M, Ferrando-Vivas P, Gore C, Power S, Harrison D, et al. Characteristics and Outcome of Cancer Patients Admitted to the ICU in England, Wales, and Northern Ireland and National Trends Between 1997 and 2013. Crit Care Med. 2017; 45:1668-76.

12. Groeger JS, Bach PB. Consider saying yes. Crit Care Med. 2003;31:320-1.

13. Raoof ND, Groeger JS. You never know--one of your patients with cancer might surprise you. Crit Care Med. 2007;35:965-6.

14. Xia R, Wang D. Intensive care unit prognostic factors in critically ill patients with advanced solid tumors: a 3-year retrospective study. BMC cancer. 2016;16:188.

15. Wallace SK, Rathi NK, Waller DK, Ensor JE Jr, Haque SA, Price KJ, et al. Two Decades of ICU Utilization and Hospital Outcomes in a Comprehensive Cancer Center. Crit Care Med. 2016; 44:926-33.

16. Wang JY, Lee MC, Shu CC, Lee CH, Lee LN, Chao KM, et al. Optimal duration of anti-TB treatment in patients with diabetes: nine or six months? Chest. 2015;147:520-8.

17. Lai CL, Wu CF, Kuo RN, , Yang YY, Chen MF, Chan KA, et al: Clinical outcomes in low risk coronary artery disease patients treated with different limus-based drug-eluting stents--a nationwide retrospective cohort study using insurance claims database. PloS one. 2015;10:e0122860.

18. Chiang CJ, You SL, Chen CJ, Yang YW, Lo WC, Lai MS. Quality assessment and improvement of nationwide cancer registration system in Taiwan: a review. Jpn J Clin Oncol. 2015;45:291-6.

19. Chen YC, Chang SC, Pu C, Tang GJ. The impact of nationwide education program on clinical practice in sepsis care and mortality of severe sepsis: a population-based study in Taiwan. PloS one. 2013;8:e77414.

20. Angus DC, Linde-Zwirble WT, Lidicker J, Clermont G, Carcillo J, Pinsky MR. Epidemiology of severe sepsis in the United States: analysis of incidence, outcome, and associated costs of care. Crit Care Med. 2001; 29:1303-10.

21. Deyo RA, Cherkin DC, Ciol MA. Adapting a clinical comorbidity index for use with ICD-9-CM administrative databases. J Clin Epidemiol. 1992;45:613-9.

22. Hsu CC, Lee $\mathrm{CH}$, Wahlqvist ML, Huang HL, Chang HY, Chen L, et al. Poverty increases type 2 diabetes incidence and inequality of care despite universal health coverage. Diabetes Care. 2012;35:2286-92.

23. Chang CT, Wu SC, Hung YN, NG YY, Chen HS. The effects of individual and neighborhood socioeconomic status on early nephrology referral for hemodialysis patients. Taiwan Journal of Public Health. 2014; 02:75-88.

24. Shrime MG, Ferket BS, Scott DJ, et al: Time-Limited Trials of Intensive Care for Critically Ill Patients With Cancer: How Long Is Long Enough? JAMA Oncol 2016;2:76-83.

25. Wright AA, Keating NL, Ayanian JZ, Lee J, Barragan-Bradford D, Pollard T, et al: Family Perspectives on Aggressive Cancer Care Near the End of Life. JAMA. 2016;315:284-92.

26. Zampieri FG, Mazza B. Mechanical Ventilation in Sepsis: A Reappraisal. Shock. 2017:47:41-46

27. Rhodes A, Evans LE, Alhazzani W, Levy MM, Antonelli M, Ferrer R, et al. Surviving Sepsis Campaign: International Guidelines for Management of Sepsis and Septic Shock: 2016. Intensive Care Med. 2017;43:304-377.

28. Coba V, Whitmill M, Mooney R, Horst HM, Brandt MM, Digiovine B, et al. Resuscitation bundle compliance in severe sepsis and septic shock: improves survival, is better late than never. J Intensive Care Med. 2011;26:304-313.

29. Koyner JL, Murray PT. Mechanical ventilation and lung-kidney interactions. Clin J Am Soc Nephrol. 2008;3:562-570.

30. Esteban A, Frutos-Vivar F, Ferguson ND, Peñuelas O, Lorente JA, Gordo F, et al. Sepsis incidence and outcome: contrasting the intensive care unit with the hospital ward. Crit Care Med .2007;35:1284-1289.

31. Vincent JL, Moreno R, Takala J, Willatts S, De Mendonça A, Bruining H, et al. The SOFA (Sepsis-related Organ Failure Assessment) score to describe organ dysfunction/failure. On behalf of the Working Group on Sepsis-Related Problems of the European Society of Intensive Care Medicine. Intensive Care Med. 1996;22:707-710.

32. Wen CP, Tsai SP, Chung WS, A 10-year experience with universal health insurance in Taiwan: measuring changes in health and health disparity. Ann Intern Med. 2008;148:258-267.

33. Cheng KC, Lu CL, Chung YC, Huang MC, Shen HN, Chen HM, et al: ICU service in Taiwan. J Intensive Care. 2014;2:8 
34. Kaukonen KM, Bailey M, Suzuki S, Pilcher D, Bellomo R. Mortality related to severe sepsis and septic shock among critically ill patients in Australia and New Zealand, 2000-2012. JAMA. 2014; 311:1308-1316.

35. Labelle A, Juang P, Reichley R, Micek S, Hoffmann J, Hoban A, et al: The determinants of hospital mortality among patients with septic shock receiving appropriate initial antibiotic treatment. Crit Care Med. 2012; 40:2016-2021.

36. Halpern NA, Pastores SM, Oropello JM, Kvetan V. Critical care medicine in the United States: addressing the intensivist shortage and image of the specialty. Crit Care Med. 2013; 41:2754-2761.

37. Truog RD, Brock DW, Cook DJ, Danis M, Luce JM, Rubenfeld GD, et al. Task Force on Values, Ethics, and Rationing in Critical Care (VERICC) Rationing in the intensive care unit. Crit Care Med. 2006; 34:958-963.

38. VanderWalde NA, Salloum RG, Liu TL, Hornbrook MC, O'Keeffe Rosetti MC, Ritzwoller DP, et al. Positron emission tomography and stage migration in head and neck cancer. JAMA otolaryngology Head Neck Surg. 2014; 140:654-661.

39. Hodgson CL, Udy AA, Bailey M, Barrett J, Bellomo R, Bucknall T, et al. The impact of disability in survivors of critical illness. Intensive Care Med. 2017; 43:992-1001.

40. Hsiao WC, Cheng SH, Yip W. What can be achieved with a single-payer NHI system: The case of Taiwan. Soc Sci Med. 2016; S0277-9536(16)30676-1. 